\title{
Static correlation lengths in QCD at high temperature and finite density
}

\author{
Owe Philipsen ${ }^{\mathrm{a} *}$ \\ ${ }^{a}$ CERN Theory Division, 1211 Geneva 23, Switzerland
}

A brief review is given of the sign problem in finite density lattice QCD and various attempts to overcome it. To date there is still no solution to this problem which would work for realistic QCD. The main focus then is on the deconfined phase, where QCD can be described by a dimensionally reduced effective action. After summarizing derivation and validity of the effective theory, it is demonstrated that it can be simulated efficiently in the presence of a chemical potential for quarks $\mu / T \lesssim 4$. Direct comparison of simulations with imaginary and real $\mu$ suggests that equilibrium plasma properties could be analytically continued from 4d QCD simulations at imaginary $\mu$.

\section{INTRODUCTION}

QCD at finite baryon density occurs in nature in two rather different regimes: i) In heavy ion collisions, whose initial state has non-zero baryon number, and in which any subsequent plasma state is a state of high temperature and low density. ii) In the core of neutron stars, which is composed of cold and very dense matter. These two situations correspond to the regions close to the axes in the tentative QCD phase diagram Fig. 11. While in the latter case some interesting

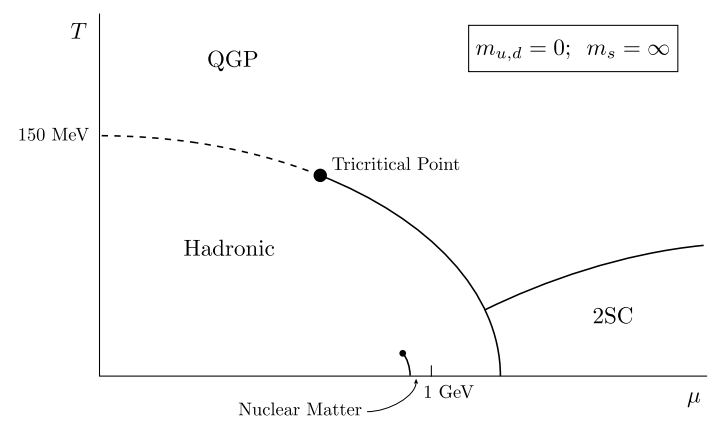

Figure 1. Conjectured phase diagram for QCD with two massless and one heavy flavour. From [1].

\footnotetext{
*Address since October 2000: Center for Theoretical Physics, Massachusetts Institute of Technology, Cambridge, MA 02139, USA
}

conjectures about a colour-superconducting state of matter have been made [2], understanding the former regime is particularly pressing in view of running and upcoming experiments at SPS, LHC (CERN) and RHIC (BNL). Eventually we would of course like to know the structure of the entire phase diagram, in particular the position and nature of the transition lines separating different regions and the (tri-) critical points.

In order to come to first principles predictions, non-perturbative methods are required. Unfortunately, lattice QCD has so far failed to be a viable tool for the analysis because of the so-called "sign-problem". The QCD partition function is given by

$$
\begin{aligned}
Z & =\int D U \operatorname{det} M(\mu) \mathrm{e}^{-S_{g}[U]}, \\
S_{f} & =\int d^{4} x \sum_{\text {colour, flavour }} \bar{\psi} M \psi \\
M & =\mathcal{D}-\mu \gamma_{0}, \quad \mathcal{D}=\gamma \cdot(\partial+\mathrm{i} A)+m,
\end{aligned}
$$

where $S_{g}$ is the pure gauge action. The relation

$\gamma_{5} M \gamma_{5}=M^{\dagger}$

implies positivity of the fermion determinant for $\mu=0$, $\operatorname{det} M(0) \geq 0$. However, for $\mathrm{SU}(3)$ and $\mu \neq 0$, Eq. (2) does not hold, $\operatorname{det} M$ is complex and prohibits standard Monte Carlo importance sampling, for which a manifestly positive measure in the functional integral is required.

In the first part of this contribution, a short review is given on the various attempts and tech- 
niques proposed in the literature to deal with this problem. Many of these try to implement a chemical potential at zero or low temperature and to obtain the "onset" value $\mu=\mu_{0}$, where the transition from a zero density state of matter to a state of finite density takes place. At zero temperature this should happen when one baryon can be made, i.e. $\mu_{0}=m_{B} / 3$. Since most of these techniques have been reviewed in detail in previous years [3, [4], this section will be rather brief.

The larger part is then devoted to the study of the plasma situation at high temperature and low density, where it was recently demonstrated that numerical simulations of static quantities at finite density are feasible in the framework of a perturbatively derived effective theory [5].

\section{NUMERICAL TECHNIQUES FOR FINITE DENSITY}

\subsection{Reweighting methods}

The Glasgow method [3] evaluates the partition function by absorbing the complex determinant into the observable, and doing the importance sampling with the positive part of the measure,

$\langle\mathcal{O}\rangle=\left\langle\frac{\mathcal{O} \operatorname{det} M(\mu)}{\operatorname{det} M(\mu=0)}\right\rangle_{\mu=0}$.

One may further expand the grand canonical partition function in the fugacity $z=\exp \mu / T$,

$Z(\mu, T, V)=\sum_{B} z^{B} Z(B, T, V)$,

where $Z_{B}$ is the canonical partition function at fixed baryon density, and compute the terms individually. However, numerical results give for the onset of baryon density the unphysical value $\mu_{0}=m_{\pi / 2}$ instead of the expected $\mu_{0}=m_{B / 3}$, which is also the pathological behaviour of the quenched theory [6]. It seems now understood that the reason for this failure is the poor overlap between the ensemble of configurations at $\mu=0$ used to compute averages by reweighting, and the correct ensemble at $\mu \neq 0$.

This situation can be improved by splitting the determinant in modulus and phase, $\operatorname{det} M=$ $|\operatorname{det} M| \exp (\mathrm{i} \phi)$, and to incorporate the modulus into the measure, while only the phase is used for reweighting [7],

$\langle\mathcal{O}\rangle=\left\langle\frac{\mathcal{O} \mathrm{e}^{\mathrm{i} \phi}}{\mathrm{e}^{\mathrm{i} \phi}}\right\rangle_{|\operatorname{det} M|}$.

The expectation value of the phase factor can be viewed as a ratio of two partition functions, one of the true theory and one with the positive modulus of the determinant in its measure. It behaves thus as $\exp -\Delta F$, where the exponent is the free energy difference and an extensive quantity. Consequently, $\langle\exp i \phi\rangle \sim \exp (V)$, which requires exponentially large statistics for realistic volumes. A similar variant of the Glasgow method works for heavy quarks [8].

\subsection{Static quarks}

The sign problem can be handled in a static quark model, in which all quarks are infinitely heavy [9. 10]. It is obtained by sending both quark mass and chemical potential to infinity $\mu, m \rightarrow$ $\infty$, while fixing a finite baryon number. This is achieved by keeping the constant

$C=(2 a m / \exp (a \mu))^{N_{t}}$

fixed, where $N_{t}$ is the temporal extent of the lattice. In this case an analytic expression for the determinant is obtained which is trivial to evaluate. The available computing time then suffices to gather large enough statistics for the phase of the determinant to be calculated with sufficient accuracy. The question is whether results obtained for static quarks translate at all to QCD with small quark masses. A more detailed discussion of this approach is presented in another contribution to this conference 11].

One may attempt to extend this approach to finite but large quark masses by going beyond the leading term in the $1 / m$-expansion, based upon an interesting observation 12]: analysis of the data of [10] shows a correlation between the phase of the fermion determinant and the imaginary part of the Polyakov line, $\phi \sim V P_{i}$, as shown in Fig. 2. Indeed, the leading order term in $1 / m$ for the phase is 12 ]

$\phi=2(2 / m)^{N_{t}} V \sinh \left(N_{t} \mu\right) P_{i}$.

The correlation is weakened by higher terms in the quark mass expansion which do not show 


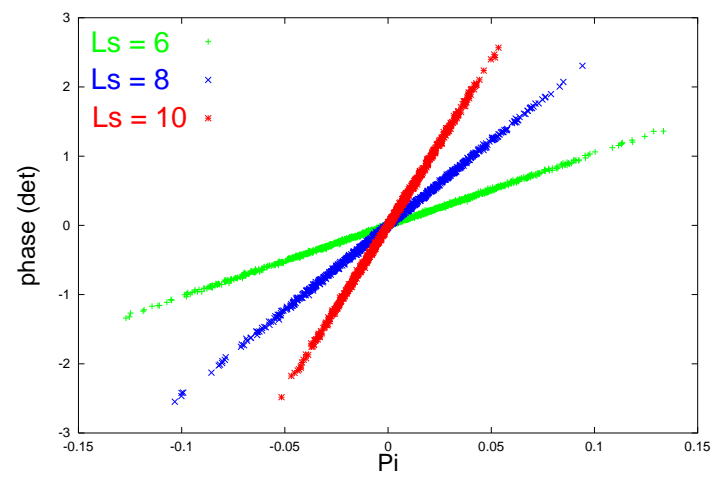

Figure 2. Correlation between the phase of the fermion determinant and the imaginary part ot the Polyakov line for static quarks at finite density on various volumes. From [12].

this proportionality, and therefore it weakens at smaller quark masses.

This suggests the following approximation: computing a constrained partition function

$Z^{\prime}=\int D U \operatorname{det} M \mathrm{e}^{-S_{g}[U]} \delta\left(P_{i}[U]\right)$

eliminates configurations with significantly fluctuating phases $\phi$ whenever its correlation with $P_{i}$ is strong, and thus improves the sign problem significantly. The approximation is good, i.e. $Z^{\prime} \approx Z$, if $P_{i} \ll P_{r}$, which is the case at small $\mu / T$, or if $P_{r} \approx P_{i} \approx 0$ at $T \sim 0, \mu<\mu_{0}$. We shall come back to this point when hot QCD at finite density is discussed.

\subsection{Imaginary chemical potential}

There are a number of suggestions to consider imaginary chemical potential, for which the integration measure is positive and simulations are straightforward. The relation to real chemical potential is provided by the canonical partition function at fixed quark number $Q$, which is related to the grand canonical partition function at fixed imaginary chemical potential by 13 .

$Z(T, Q)=\int_{-\pi T}^{+\pi T} \frac{d \mu_{I}}{2 \pi T} Z\left(T, \mu=\mathrm{i} \mu_{I}\right) e^{-i \mu_{I} Q / T} .(9)$
One proposal is to simulate $Z$ for various values of $\mu_{I}$, and then numerically do the Fourier integration [14. This becomes more and more difficult, however, for large $Q$, and extrapolation to the thermodynamic limit seems questionable. The method has been tested in the two-dimensional Hubbard model [14], but not for QCD.

A second proposal is to compute expectation values with the grand canonical partition function $Z(\mathrm{i} \mu, T)$, fit them by power series in $\mu$ and analytically continue the series to real $\mu$. This has been explored for the chiral condensate in the strong coupling limit [15].

Finally, starting from Eq. (9), a well-defined quenched limit of the theory at finite density can be obtained [16]. The limit $m \rightarrow \infty$ is taken in the expression Eq. (9), upon which it goes over into

$Z(T, Q)=\int D U f_{Q}[P] \mathrm{e}^{-S_{g}[U]}$,

where $f_{Q}[P]$ is a functional of Polyakov lines. The quenched limit thus is not simply realized by replacing the fermion determinant by a constant. Instead, there are remainders of the static quark propagators in the partition function, the Polyakov loops, which carry the baryon number. The expression after the limit is still complex, and thus continues to have a sign problem. However, simulations have shown that it is weakened enough to be well manageable numerically, and results for the static potential are available [16].

\subsection{Avoiding the sign problem: $\mathrm{SU}(2)$}

In view of the difficulties with $\mathrm{SU}(3)$, one may resort to studying two colour QCD which, thanks to the realness of the $\mathrm{SU}(2)$ matter representations, has a real and positive fermion determinant and can be simulated straightforwardly [17. Unfortunately, the same property leads to other peculiarities which make it less analogous to QCD than one might have hoped: charge conjugation is related to global SU(2) rotations. Thus, the condensates $\langle q \bar{q}\rangle$ and $\langle q q\rangle$ are related by rotations as well, and there is no fundamental distinction between them. Since there are only two colours, 'baryons' consist of even numbers of quarks, and through $\mathrm{SU}(2)$ rotations they are equivalent to 
mesons. In particular, this results in a physical onset chemical potential $\mu_{0}=m_{\pi} / 2$, even in the unquenched theory. Moreover, since $\langle q q\rangle$ is gauge-invariant, there can be no colour superconductivity either. Hence, the model is simulable, but some main questions of interest for QCD have disappeared.

Somewhat surprisingly, these questions reenter if the representation of the fermions is taken to be adjoint and only one flavour is considered [18]. In this case, there are no $q q$ Goldstone modes. Moreover, the theory permits gauge-invariant threequark baryon operators $q q q$ with spin $1 / 2$, and thus $\mu_{0}$ for the finite density onset should be different from half the meson mass. Further, $\langle q q\rangle$ is gauge-variant and hence permits a study of colour superconductivity. Finally, in the lattice model with an adjoint fermion, the determinant is real but not positive definite, hence a weakend sign problem is present which can be handled by a two-step multi-boson algorithm [18].

More detailed discussions of $\mathrm{SU}(2)$ models together with numerical results may be found in the finite density section of these proceedings 19 .

\subsection{Finite isospin density}

In has also been proposed to consider a chemical potential for isospin rather than for baryon number 14 20]. In this case, and with two degenerate light flavours $m_{u}=m_{d}$, Eq. (2) can be supplemented by an isospin rotation to give

$\tau_{1} \gamma_{5} M \gamma_{5} \tau_{1}=M^{\dagger}$,

which again implies $\operatorname{det} M \geq 0$ and thus can be simulated. Analytic work for very small and large isospin chemical potential, where chiral perturbation theory or ordinary perturbation theory are applicable, respectively, lead to the conjecture that the hadron and quark matter phases are smoothly connected 20 .

\subsection{Hamiltonian formulation}

Another attempt addresses the problem in a Hamiltonian formulation of lattice QCD at finite density [21]. Quark number density, chiral condensate, some meson and the nucleon masses have been calculated in the limits of a free and a strongly coupled theory at zer temperature. The chiral phase transition is found to be first order, and the onset $\mu_{0}$ is consistent with $m_{B} / 3$. So far, however, no numerical implementation of this approach is available.

\subsection{Cluster algorithm}

Finally, a new algorithm has been proposed to actually solve the sign problem [22]. So far, it has only been worked out for non-relativistic spin systems. The approach uses cluster algorithms to decompose a fermion configuration into clusters of spins that can be flipped independently, individually changing sign or not under the flip. This enables to cancel negative against positive contributions, leaving only net positive contributions. Details and possible extensions to QCD are discussed in the contribution [11].

\subsection{Finite temperature and density}

All proposals that have been implemented numerically have thus failed on realistic QCD so far, and the failure is particularly drastic at low temperatures. The methods that do work numerically are limited to static or very heavy fermions, and they work better at high temperatures, suggesting that the sign problem may be easier to overcome in hot QCD. Let us then shift attention to temperatures above the deconfinement transition.

\section{EFFECTIVE THEORY APPROACH TO HOT QCD}

For temperatures larger than a few times the deconfinement temperature $T_{c}$, the static (equilibrium) physics of QCD can be described by an effective theory, which indeed permits simulations with non-vanishing real chemical potential [5]. Of course, the restriction to the deconfined phase presents a limitation. Another one is that finite density simulations are possible only up to some value of the chemical potential, where the sign problem sets in. Presently the accessible range is $\mu \lesssim 4 T$. Finally, if the effective theory is to be quantitatively accurate, one has to be sufficiently away from the deconfinement transition.

If there are limitations, there are also strengths of this approach. The effective theory is threedimensional and purely bosonic, so simulations 
are cheap and yield rather precise continuum limits, leaving little ambiguity in the interpretation of the results. The approach is good for $\mathrm{SU}(2)$ as well as $\mathrm{SU}(3)$, and permits to study any number of quarks with any small or zero mass. Finally, the domain of parameter space where the approach is applicable contains the phenomenologically relevant region in which heavy ion collisions are operating. At and above SPS energies, densities in heavy ion collisions are estimated to be $\mu_{B} / T \lesssim 4.0$ [23], i.e. a quark chemical potential $\mu / T \lesssim 1.3$, which is well within the range where simulations are feasible.

\subsection{Equilibrium correlation lengths}

Let us first discuss the physics question that will be addressed in the following. Consider gauge-invariant, local operators $A(x)$. Static equilibrium physics is described by euclidean time averages

$\bar{A}(\mathbf{x})=T \int_{0}^{1 / T} d \tau A(\mathbf{x},-\mathbf{i} \tau)$.

The spatial correlation function of such operators,

$C(|\mathbf{x}|)=\langle\bar{A}(\mathbf{x}) \bar{A}(0)\rangle_{c} \sim \mathrm{e}^{-M|\mathbf{x}|}$,

falls off exponentially with distance. The "screening masses" $M$ have a precise non-perturbative definition: they are the eigenvalues of the spacewise transfer matrix in the corresponding lattice field theory. Physically, they correspond to the inverse length scale over which the equilibrated medium is sensitive to the insertion of a static source carrying the quantum numbers of $A$. Beyond $1 / M$, the source is screened and the plasma appears undisturbed. With these definitions, all screening lengths corresponding to gauge-invariant sources can be computed on the lattice in principle.

\subsection{Dimensional reduction}

If we are interested in length scales larger than the inverse temperature, $|\mathbf{x}| \sim 1 / g T \gg 1 / T$, the situation simplifies considerably. In this case the integration range for euclidean time averaging in Eq. (12) becomes very small, and the problem effectively three-dimensional (3d).

The calculation of the correlation function $C(|\mathbf{x}|)$ can be factorized: the time averaging may be performed perturbatively by expanding in powers of the ratio of scales $g T / T \sim g$, which amounts to integrating out all modes with momenta $\sim T$ and larger, i.e. all non-zero Matsubara modes, in particular the fermions. This procedure is known as dimensional reduction [24]. It is in the spirit of a Wilsonian renormalization group approach, where an effective action for coarser scales is derived by averaging over the smaller scales. However, here this step is done perturbatively and entails two approximations: the first is that the computation is obviously limited to a finite order in $g$. The second is to neglect higherdimensional operators, which are suppressed by powers of the scale ratio?.

The remaining correlation function of $3 \mathrm{~d}$ fields is then to be evaluated with a $3 \mathrm{~d}$ purely bosonic effective action, which describes the physics of the modes $\sim g T$ and softer. The screening masses $M$ now correspond to the eigenvalues of the $3 \mathrm{~d}$ transfer matrix. Simulation of this reduced problem is easy. Without fermions and one dimension less, much larger volumes and finer lattices can be considered. Moreover, 3d gauge theories are superrenormalizable and the coupling scales linearly with lattice spacing. Hence, very accurate continuum limits can be obtained.

The effective theory emerging from hot QCD by dimensional reduction is the $\mathrm{SU}(3)$ adjoint Higgs model 24,26.27 with the action

$$
\begin{aligned}
S=\int d^{3} x & \left\{\frac{1}{2} \operatorname{Tr}\left(F_{i j} F_{i j}\right)+\operatorname{Tr}\left(D_{i} A_{0}\right)^{2}\right. \\
& +m_{3}^{2} \operatorname{Tr}\left(A_{0}^{2}\right)+\lambda_{3}\left(\operatorname{Tr}\left(A_{0}^{2}\right)^{2}\right\} .
\end{aligned}
$$

As $4 \mathrm{~d}$ euclidean time has been integrated over, $A_{0}$ now appears as a scalar in the adjoint representation. The associated Hamiltonian respects $\mathrm{SO}(2)$ planar rotations, two-dimensional parity $P$, charge conjugation $C$ and $A_{0}$-reflections $R$.

The parameters of the effective theory are via dimensional reduction functions of the $4 \mathrm{~d}$ gauge coupling $g^{2}$, the number of colours $N$ and flavours $N_{f}$, the fermion masses and the temperature $T$. In all of the following fermion masses are assumed

\footnotetext{
${ }^{2} \mathrm{~A}$ method to construct an effective theory by a (perturbative) block spin transformation has been proposed in [25.)
} 
to be zero, but in principle any other values may be considered as well. At leading order in the reduction step one has

$$
\begin{aligned}
g_{3}^{2} & =g^{2}(\bar{\mu}) T, \\
m_{3} & =\left(\frac{N}{3}+\frac{N_{f}}{6}\right)^{1 / 2} g(\bar{\mu}) T, \\
\lambda_{3} & =\frac{1}{24 \pi^{2}}\left(6+N-N_{f}\right) g^{4}(\bar{\mu}) T .
\end{aligned}
$$

Note that $m_{3}$ is just the leading order Debye mass [28]. The reduction step has been performed up to two-loop order 27] at which parameters have relative accuracy $\mathcal{O}\left(g^{4}\right)$ and 1-loop scale dependence on $\bar{\mu}$ is cancelled. Specifying $T / \Lambda_{\overline{\mathrm{MS}}}, N_{f}$ then completely fixes the effective theory.

If we are interested in the infrared-sensitive, non-perturbative scales of order $\sim 1 / g^{2} T$ 29, the fields $A_{0} \sim g T$ may be integrated out as well, leaving us with a $3 \mathrm{~d}$ pure gauge theory. In order to decide which is the correct effective theory for a given temperature beyond parametric considerations, simulation results for screening lengths will be compared between the reduced and the full theory.

With the simulation part being rather accurate, the main error in the correlation functions is due to the reduction step, and at two-loop level this is formally of the order 31]

$\frac{\delta C}{C} \sim \mathcal{O}\left(g^{3}\right)$.

In the treatment of the electroweak phase transition, this is much less than 5\% [30], but for hot QCD the coupling and thus the error is larger. In fact, one may wonder why perturbative reduction should work at all, given that the effective $g^{2} \sim 2.7$ at $T=2 T_{c}$ [27]. In the loop expansion, the coupling appears as $\alpha_{s} / \pi$ rather than $g$. Inspecting the computed series for the effective theory parameters Eq. (15) up to next-to-leading order, it appears that it converges surprisingly well at temperatures not much above the critical one [27,32. The other source of error is neglecting higher dimension operators. This is justified as long as the dynamical mass scales of the effective theory are smaller than those integrated out $\sim 2 \pi T$. The success of the approach then
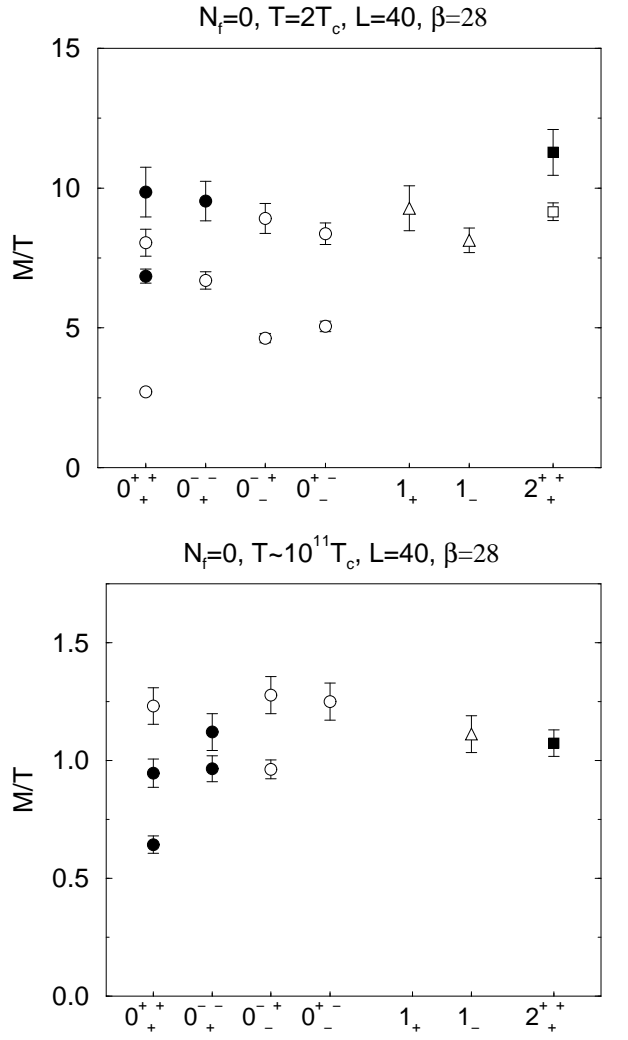

Figure 3. Spectrum of screening masses in various quantum number channels at low and high temperatures, for $\mathrm{SU}(3), N_{f}=0$. Filled symbols denote $3 \mathrm{~d}$ glueball states. From 5 .

depends on whether there is a non-perturbative scale separation. This can only be checked after simulating the effective theory.

\subsection{Numerical results}

Results from simulations [5] of the gaugeinvariant screening spectrum with the effective theory Eq. (14) are displayed in Fig. 3. Screening masses are classified according to the symmetries of the 3d Hamiltonian by the quantum numbers $J_{R}^{P C}$. The open symbols show states dominated by scalar operators such as $\operatorname{Tr}\left(A_{0}^{2}\right), \operatorname{Tr}\left(A_{0} F_{12}^{2}\right)$ etc., whereas full symbols show states receiving only gluonic contributions $\operatorname{Tr}\left(F_{12}^{2}\right)$ etc. It is a remarkable finding of detailed mixing analyses in several models [33, 34, 5], that the latter are quan- 
titatively consistent with the $3 \mathrm{~d}$ glueball states 35 and apparently completely insensitive to the presence of the $A_{0}$ scalar field. Because of this pronounced non-mixing, we can thus state a first important result: For any reasonable temperatures the largest correlation length of gaugeinvariant operators belongs to the $A_{0}$ degrees of freedom and not to the $A_{i}$, in contrast to the naive parametric picture. This demonstrates that the physics from the scale $g T$ down to $g^{2} T$ is completely non-perturbative, and hence $A_{0}$ may not be integrated out perturbatively. Only for temperatures $T \gtrsim 10^{7} T_{c}$ becomes the coupling small enough that the parametrically expected ordering of screening masses is realized.

\subsection{Testing dim. red. in hot Yang-Mills}

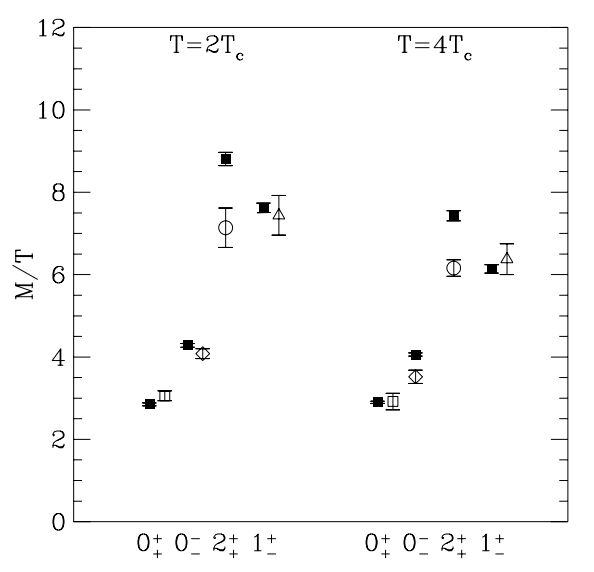

Figure 4. Comparison of screening masses $J_{R}^{P}$ $(C=+)$ in hot $\mathrm{SU}(2)$ pure gauge theory as determined in $4 \mathrm{~d}$ (empty symbols) and 3d (full symbols) effective theory simulations. From [34].

We now wish to know the accuracy of the reduced theory. Fig. 1 compares the results for hot $\mathrm{SU}(2)$ gauge theory as obtained in the 4d lattice theory [36] with those from the effective theory [34]. Note that the effective theory is only valid up to its cut-off $M / T \sim 2 \pi$ and above this level disagreement is to be expected. The apparent excellent agreement for the two lowest lying states should be taken with some care given some
Table 1

Comparison of the lowest two screening masses in SU(3) Yang-Mills at $T=2 T_{c}$, obtained in 3d [5] and $4 \mathrm{~d}[36]$ simulations.

\begin{tabular}{|c|c|c|}
\hline$M / T$ & $3 \mathrm{~d}$ & $4 \mathrm{~d}$ \\
\hline $0_{+}^{++}$ & $2.71(6)$ & $2.60(4)$ \\
\hline $0_{-}^{-+}$ & $4.6(2)$ & $6.3(2)$, \\
\hline
\end{tabular}

systematic uncertainties. In $4 \mathrm{~d}$ simulations, the projection properties of operators and the scaling behaviour are more difficult to control than in $3 \mathrm{~d}$. In the effective theory, there is an ambiguity in the choice of renormalization scale. The direct comparison may thus still suffer from effects up to $20 \%$. For the case of SU(3) one finds again quantitative agreement in the largest correlation length but indeed about $20 \%$ deviation in the next shorter one, cf. Table f. Good agreement between the $4 \mathrm{~d}$ and the $3 \mathrm{~d}$ effective theory is also found with other observables, like the static potential [26], the spatial string tension [37], the Debye mass [38] and gauge-fixed propagators [39. [3]

Thus, dimensionally reduced pure gauge theory gives at least a semi-quantitative description of the largest correlation lengths in the system down to temperatures as low as $\sim 2 T_{c}$.

\subsection{Inclusion of fermions}

The main advantage of the effective theory is that fermions, having always non-zero Matsubara frequency, are treated analytically. Any change in the number of fermion species or their masses is encoded in the parameters of the effective theory, Eq. (15). The results for the spin zero channels at various $N_{f}$ (all massless) are shown in Fig. 河. The screening masses get larger with increasing $N_{f}$, so that even the smallest of them comes close to the cut-off $\sim 2 \pi T$ of the effective theory, thus diminishing the scale separation needed for the effective theory to be valid. Consequently, only the lowest state may be expected to describe hot 4 d physics. As in the $N_{f}=0$ case, this situation is improved by going to larger temperatures.

Another, and more severe, limitation comes

${ }^{3}$ Dimensional reduction also works to high precision from 3 d finite $T$ to $2 \mathrm{~d} 40$. 


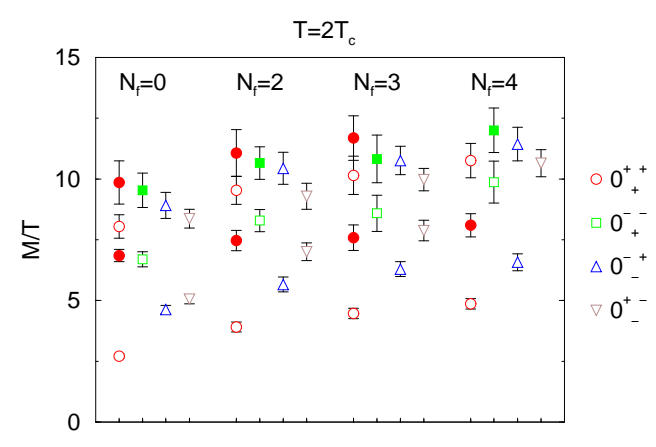

Figure 5. Spectrum of scalar screening masses for various $N_{f}$ with zero fermion masses. Filled symbols denote 3 d glueball states. From [0].

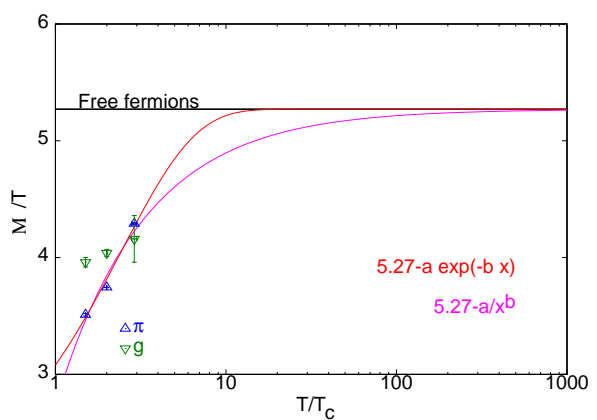

Figure 6. The smallest scalar screening masses due to correlations of gluonic (g) and mesonic $(\pi)$ operators. The lines represent phenomenological fits. From 41.

from the fact that, close to $T_{c}$, the fermionic modes begin to feel the chiral phase transition and thus become very light through nonperturbative effects. At some point this effect will be so large that they constitute the lightest degrees of freedom and may no longer be integrated out. This situation has been demonstrated recently in a $4 \mathrm{~d}$ simulation with $N_{f}=4$ light fermions [41], and is shown in Fig. 6. Below $T \sim 2-3 T_{c}$, the pion correlator yields the longest correlation length, and the purely bosonic effective theory fails to give the correct infrared behaviour. This problem becomes worse for larger $N_{f}$, whereas it is slightly better for the realistic case of two light fermion flavours. It would be very interesting to see if this behaviour can be reproduced in the effective theory, if NRQCD methods are applied and fermionic degrees of freedom are kept in the effective action [42].

\section{DIMENSIONAL REDUCTION AT FI- NITE DENSITY}

Having seen that dimensionally reduced QCD gives a reasonable description for the longest correlation length at temperatures $T \gtrsim 2-3 T_{c}$, we may now return to the problem of introducing a chemical potential [5]. The contribution of the fermion determinant with $\mu \neq 0$ to the effective potential of $A_{0}$ has been evaluated at the twoloop level [43]. From this one may derive the corrsponding changes in the three-dimensional effective action, brought about by the chemical potential term. To leading order, it generates one extra term in the action Eq. (14) and changes the bare $A_{0}$ mass parameter,

$$
\begin{aligned}
S & \rightarrow S+\mathrm{i} z \int d^{3} x \operatorname{Tr} A_{0}^{3}, \quad z=\frac{\mu}{T} \frac{N_{f}}{3 \pi^{2}}, \\
m_{3} & \rightarrow m_{3}\left[1+\left(\frac{\mu}{\pi T}\right)^{2} \frac{3 N_{f}}{2 N+N_{f}}\right] .
\end{aligned}
$$

The extra term $S_{z}$ is odd under $R, C$, and hence the action no longer respects these symmetries, while parity is left intact. Consequently, screening states now are labelled by $J^{P}$.

The effective action is complex just as the $4 \mathrm{~d}$ original one, and hence there is a sign problem. Expectation values have to be computed by reweighting with the complex piece of the action

$\langle\mathcal{O}\rangle=\frac{\left\langle\mathcal{O} \mathrm{e}^{-\mathrm{i} z S_{z}}\right\rangle_{0}}{\left\langle\cos \left(z S_{z}\right)\right\rangle_{0}}$,

where the subscript denotes averaging with $\mu=0$. Cancelling contributions to the expectation value occur whenever $\left(z S_{z}\right) \gg 1$. The perturbative width of the distribution at two-loop level can be computed from the $A_{0}$ sunset diagram to give

$$
\begin{aligned}
& \Delta\left(z S_{z}\right) \approx \frac{z}{4 \pi}\left(\frac{6 L}{\beta}\right)^{3 / 2} \\
& \times\left[5\left(\ln \frac{1}{12 a m_{D}}+0.58849\right)\right]^{1 / 2} .
\end{aligned}
$$



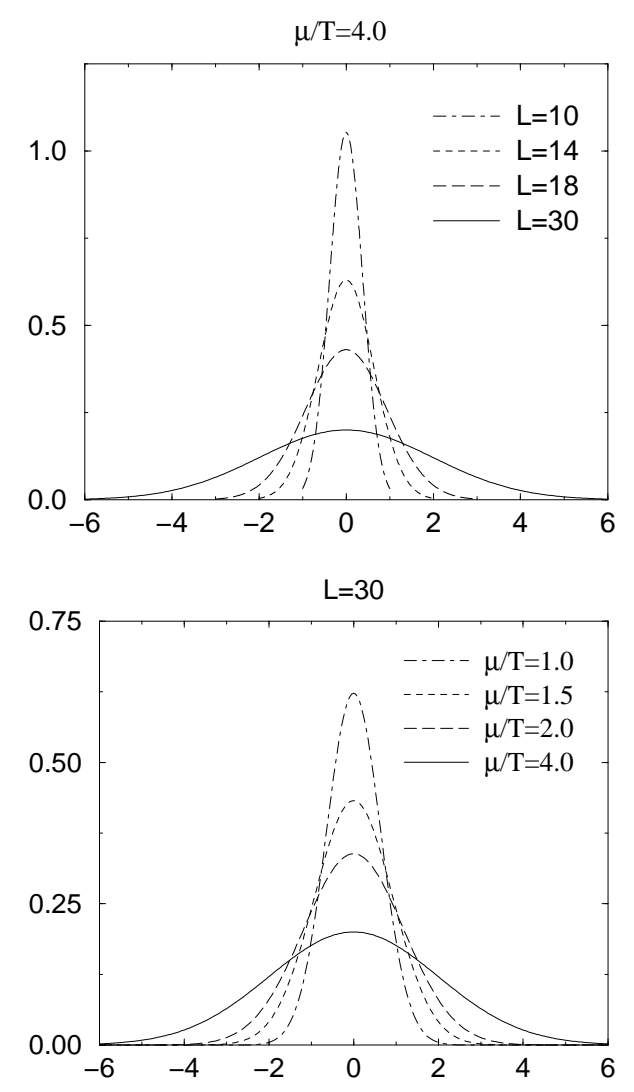

Figure 7. Distribution of the reweighting factor $\left(z S_{z}\right)$ for fixed $\mu / T$ and various $L$ (top), and for fixed $L$ and various $\mu / T$ (bottom). From [5].

One recognizes the same behaviour as in the $4 \mathrm{~d}$ case, in that the width of the phase distribution is growing with $\sim \sqrt{V}(\mu / T)$. Fig. 7 shows some distributions of $\left(z S_{z}\right)$ as obtained by Monte Carlo for various volumes and chemical potentials. As long as $\mu \lesssim 4 T$, the distribution is well contained within $[-\pi, \pi]$ for volumes large enough so that the masses attain their infinite volume values [5]. Correspondingly, as long as this condition is met, there are no problems with cancelling contributions to correlation functions, and the signal is as good as at $\mu=0$. For even larger values of the chemical potential the sign problem sets in and statistical errors explode.

Next, one has to check the overlap between the
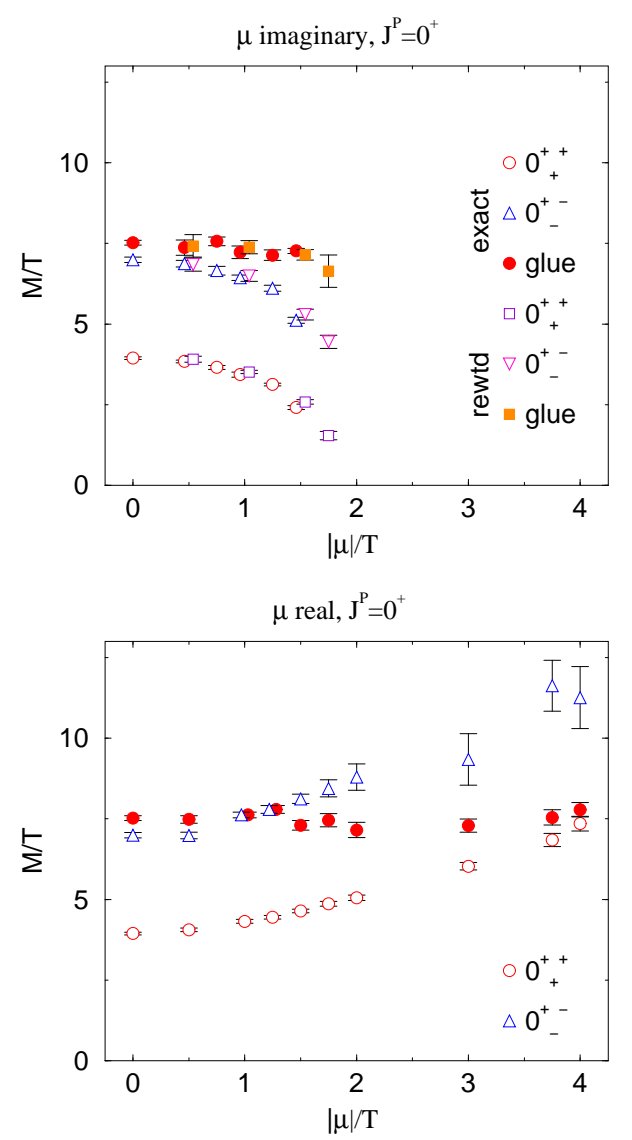

Figure 8. The lowest scalar screening masses when an imaginary (top) or real (bottom) chemical potential is switched on. From [5].

ensembles at $\mu \neq 0$ and $\mu=0$ in order to avoid a wrong biasing of the importance sampling by the reweighting procedure. For a given $|\mu| / T$, one can get an idea about this using imaginary chemical potential, where reweighting can be compared with the full action update. This is shown in the top panel of Fig. 8. Within the small statistical errors, no difference between reweighted and original ensembles is noticable. This result then gives us confidence in the corresponding ones obtained for real chemical potential, bottom of Fig. ․

Because of the different qualitative behaviours of $3 \mathrm{~d}$ gluonic and scalar states mentioned earlier, we may expect to observe a change in the nature of the ground state excitation at some real 
value of $\mu$. Indeed, Fig. 8 bottom suggests a level crossing at $\mu / T \sim 4.0$. This would mean that the longest correlation length in the thermal system does not get arbitrarily short with increasing density, but rather stays at a constant level. Note that the value of $M / T$ at this crossing is already so large that the effective theory may be inaccurate quantitatively. However, the qualitative effect should be the same in the $4 \mathrm{~d}$ theory.

\section{RELATION TO $4 \mathrm{D}$}

One may now ask why the sign problem in the effective theory is weakened, and what the relation to the original problem in four dimensions is. To this end, note that the fermionic Matsubara modes at finite temperature have propagators $\left(p_{i} \gamma_{i}+m+\gamma_{0}(2 n+1) \pi T\right)^{-1}$. For large $T$ these modes are heavy, which is why dimensional reduction integrates them out. We can then make contact to the heavy quark expansion of Sec. 2.2, where a correlation between the phase of the fermion determinant and the imaginary part of the Polyakov loop has been observed (cf. Fig. 2). Expanding the exponential of the imaginary part of the Polyakov loop $P_{i} \sim \operatorname{Im} \operatorname{Tr}\left(\int d \tau A_{0}\right)^{3}+\ldots$ indicates that after integrating over euclidean time the leading contribution is

$\phi \sim \frac{\mu}{T} S_{z}$,

which is just the extra term in the effective action Eq. (17). There, part of the path integral has been computed already analytically, namely the integration over the hard modes of order $p \geq T$. This has produced the numerical factor in $z$, Eq. (17, 19), suppressing the width of the distribution relative to the one before this integration.

\section{IMAGINARY VS. REAL CHEMICAL POTENTIAL}

With an effective theory permitting simulation of real and imaginary chemical potential at hand, one may now return to an earlier suggestion and study the feasibility of analytic continuation of observables from imaginary to real $\mu$ [44]. Away from phase transitions, the screening masses have to be analytic in $\mu / T$, as there are no massless
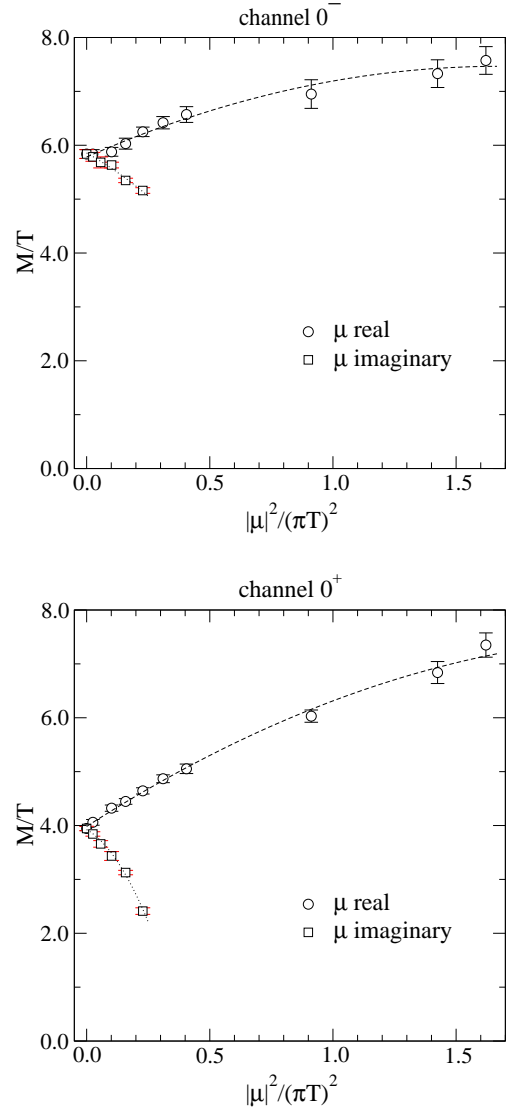

Figure 9. Fits of Eq. (21) to the lowest two screening masses at imaginary and real chemical potential. From 44.

modes in the theory. Moreover, since a change $\mu \rightarrow-\mu$ can be compensated for by a field redefinition $A_{0} \rightarrow-A_{0}$ in Eq. (14), all physical observables must be even under this operation. In the original $4 \mathrm{~d}$ theory the same statement follows from compensating $\mu \rightarrow-\mu$ by a $\mathrm{C}$ (or $\mathrm{CP}$ ) operation. For small values of $\mu / T$, the screening masses may thus be written as

$$
\frac{M}{T}=c_{0}+c_{1}\left(\frac{\mu}{\pi T}\right)^{2}+c_{2}\left(\frac{\mu}{\pi T}\right)^{4}+\mathcal{O}\left(\frac{\mu}{\pi T}\right)^{6} .
$$

One may now ask to what extent a truncated power series like this reproduces the data. In the range where this is possible for both real and imaginary $\mu$, the possibility of analytic continua- 
tion is easily checked by examining whether the $c_{i}$ agree between the two data sets. Fig. 9 shows the lowest states in the system for real and imaginary potential, together with the fits to Eq. (21).

Note that in the imaginary $\mu$ case, data are only available up to $|\mu| / T \sim 1.5$, and hence $c_{2}$ is not well constrained. The reason is that imaginary chemical potentials favour a $Z_{N}$-broken minimum over the symmetric one in the $4 \mathrm{~d}$ effective $A_{0}$ potential, once $|\mu| / T \geq \pi / 3$. Thus a phase transition occurs and analyticity is lost. In the effective theory, this transition shows up by a decrease of the scalar bare mass parameter sending the system into a broken phase. In the region up to this critical value, however, complete compatibility for the values of $c_{0,1}$ is observed between the two data sets 144 .

One may object that, once the deconfinement transition line is continued to complex values of $\mu$, it may get distorted, and a continuation might fail if it pierces the transition sheet $T_{c}(\mu)$ while rotating from imaginary to real values. In fact, for imaginary $\mu$ screening masses are decreasing. Correspondingly we would expect the line $T_{c}(\mu)$ in the $\{T, \operatorname{Im}(\mu)\}$-plane to be an increasing function of $|\mu|$, which is the distortion we were worried about. However, the change in $T_{c}(\mu)$ should be of the same order in $\mu /(\pi T)$ as the change in the screening masses. For the maximal $|\mu| / T \leq 1.3$ allowed, the change should not be more than $20 \%$, and the procedure is surely safe for $T \gtrsim 1.5 T_{c}$.

In the region where the effective theory is applicable, it is thus found that direct analytic continuation does seem to provide a working tool for determining correlation lengths. This suggests that in $4 \mathrm{~d}$ simulations analytic continuation from imaginary chemical potential would give physical results if a good ansatz for the $\mu$-dependence is available, and would allow to go closer to $T_{c}$ determining, e.g., the free energy density and the spatial correlation lengths there.

\section{CONCLUSIONS}

In summary, numerical simulations of $\mathrm{SU}(3)$ QCD at finite density are feasible only for static or heavy quarks, where the sign problem persists but enough computational power is available to calculate the phase of the fermion determinant with sufficient accuracy. However, all numerical approaches so far fail for realistic QCD.

Qualitative insight may be gained from simulations of two-colour QCD, where the model with one flavour of adjoint fermions shares several features with QCD, or from simulations of QCD at finite isopsin density, in which cases there is no sign problem. It will be interesting to see further developments of another approach, which attempts to actually solve the sign problem by use of cluster algorithms.

At temperatures above the deconfinement transition, the equilibrium physics of $\mathrm{QCD}$ is described by a perturbatively derived, threedimensional and purely bosonic effective theory, which is the more accurate the higher the temperature. Reasonable results for the longest correlation length may be obtained for temperatures $\gtrsim 2-3 T_{c}$.

The effective theory can be efficiently simulated even after introduction of a finite chemical potential for light or massless quarks, as long as $\mu \lesssim 4 T$. This domain of applicability covers the parameter values which are phenomenologically relevant for heavy ion collision experiments. It is furthermore found that screening masses can be computed by analytic continuation of calculations at imaginary chemical potential. This points to the possibility that static plasma properties could be computed in four-dimensional QCD simulations employing imaginary chemical potential, thus circumventing the sign problem. Another problem for future work might be to improve the effective theory by NRQCD methods, allowing also hadronic observables to be computed.

\section{REFERENCES}

1. K. Rajagopal, Nucl. Phys. A661, 150 (1999).

2. D. Bailin and A. Love, Phys. Rept. 107, 325 (1984); M. Alford et al., Phys. Lett. B422, 247 (1998); R. Rapp et al., Phys. Rev. Lett. 81, 53 (1998); M. A. Halasz et al., Phys. Rev. D58, 096007 (1998); M. Alford et al., Nucl. Phys. B537, 443 (1999).

3. I. M. Barbour et al., Nucl. Phys. Proc. Suppl. 60A, 220 (1998). 
4. M. Alford, Nucl. Phys. Proc. Suppl. 73, 161 (1999); F. Karsch, Nucl. Phys. Proc. Suppl. 83, 14 (2000).

5. A. Hart, M. Laine and O. Philipsen, Nucl. Phys. B 586 (2000) 443.

6. I. Barbour et al., Nucl. Phys. B275, 296 (1986); M. A. Stephanov, Phys. Rev. Lett. 76, 4472 (1996).

7. A. Vladikas, Nucl. Phys. B (Proc. Suppl.) 4 (1988) 322; D. Toussaint, Nucl. Phys. Proc. Suppl. 17, 248 (1990).

8. R. Aloisio et al., Phys. Rev. D61, 111501 (2000).

9. I. Bender et al., Nucl. Phys. Proc. Suppl. 26, 323 (1992).

10. T. C. Blum et al., Phys. Rev. Lett. 76, 1019 (1996).

11. S. Chandrasekharan, these proceedings.

12. P. de Forcrand and V. Laliena, Phys. Rev. D61, 034502 (2000).

13. D. E. Miller and K. Redlich, Phys. Rev. D35, 2524 (1987); A. Hasenfratz and D. Toussaint, Nucl. Phys. B371, 539 (1992).

14. M. Alford, A. Kapustin and F. Wilczek, Phys. Rev. D 59 (1999) 054502.

15. M.-P. Lombardo, Nucl. Phys. B (Proc. Suppl.) 83 (2000).

16. J. Engels et al., Nucl. Phys. B558, 307 (1999).

17. E. Dagotto, F. Karsch and A. Moreo, Phys. Lett. B169, 421 (1986); S. Hands et al., Nucl. Phys. B558, 327 (1999); J. B. Kogut et al., Nucl. Phys. B582, 477 (2000).

18. S. Hands et al., hep-lat/0006018.

19. these proceedings: S. Muroya, A. Nakamura and C. Nonaka; S. J. Hands, J. B. Kogut, S. E. Morrison and D. K. Sinclair; E. Bittner, M. Lombardo, H. Markum and R. Pullirsch; S. Hands, I. Montvay, S. Morrison, M. Oevers, L. Scorzato and J. Skullerud.

20. D. T. Son and M. A. Stephanov, hepph/0005225.

21. E. B. Gregory et al., Phys. Rev. D62, 054508 (2000).

22. S. Chandrasekharan and U. Wiese, Phys. Rev. Lett. 83, 3116 (1999).

23. J. Cleymans and K. Redlich, Phys. Rev. C 60 (1999) 054908.
24. P. Ginsparg, Nucl. Phys. B170 (1980) 388; T. Appelquist and R. D. Pisarski, Phys. Rev. D23 (1981) 2305.

25. U. Kerres, G. Mack and G. Palma, Nucl. Phys. B467, 510 (1996).

26. S. Nadkarni, Phys. Rev. Lett. 60 (1988) 491; T. Reisz, Z. Phys. C 53 (1992) 169; L. Kärkkäinen et al., Phys. Lett. B 282 (1992) 121; Nucl. Phys. B 418 (1994) 3; Nucl. Phys. B 395 (1993) 733.

27. K. Kajantie et al., Nucl. Phys. B503 (1997) 357.

28. M. Le Bellac, Thermal Field Theory, Cambridge University Press, 1996.

29. A. D. Linde, Phys. Lett. B96 (1980) 289; D. J. Gross, R. D. Pisarski and L. G. Yaffe, Rev. Mod. Phys. 53 (1981) 43.

30. M. Laine, JHEP 9906, 020 (1999).

31. K. Kajantie et al., Nucl. Phys. B458 (1996) 90; Phys. Lett. B 423 (1998) 137.

32. E. Braaten and A. Nieto, Phys. Rev. Lett. 76 (1996) 1417; Phys. Rev. D 53 (1996) 3421.

33. O. Philipsen, M. Teper and H. Wittig, Nucl. Phys. B 469 (1996) 445; Nucl. Phys. B528 (1998) 379.

34. A. Hart and O. Philipsen, Nucl. Phys. B572, 243 (2000).

35. M. J. Teper, Phys. Rev. D59 (1999) 014512.

36. S. Datta and S. Gupta, Phys. Lett. B471, 382 (2000); Nucl. Phys. B534, 392 (1998).

37. G. S. Bali et al., Phys. Rev. Lett. 71, 3059 (1993); F. Karsch, E. Laermann and M. Lutgemeier, Phys. Lett. B346, 94 (1995).

38. M. Laine and O. Philipsen, Nucl. Phys. B 523 (1998) 267; Phys. Lett. B 459 (1999) 259.

39. F. Karsch, M. Oevers and P. Petreczky, Phys. Lett. B442, 291 (1998); A. Cucchieri, F. Karsch and P. Petreczky, hep-lat/0004027.

40. P. Bialas et al., Nucl. Phys. B 581 (2000) 477. 41. R. V. Gavai and S. Gupta, Phys. Rev. Lett. 85, 2068 (2000).

42. S. Huang and M. Lissia, Nucl. Phys. B 480 (1996) 623.

43. C. P. Korthals-Altes, R.D. Pisarski and A. Sinkovics, Phys. Rev. D61, 056007 (2000). 44. A. Hart, M. Laine and O. Philipsen, heplat/0010008. 